\title{
La relación entre la confianza y el compromiso y sus efectos en la lealtad de marca
}

\author{
VARGAS ROCHA, FREDERICO RAFAEL \\ Departamento de Economía de la Empresa \\ Universidad Rey Juan Carlos \\ Correo electrónico: frechavargas@hotmail.com \\ DE ESTEBAN CURIEL, JAVIER \\ Departamento de Economía de la Empresa \\ Universidad Rey Juan Carlos \\ Correo electrónico: javier.deesteban@urjc.es \\ MOURA CUNHA, LUIZ RODRIGO \\ Departamento de Economía de la Empresa \\ Fundação Pedro Leopoldo (Brasil) \\ Correo electrónico: luizrcmoura@gmail.com
}

\begin{abstract}
RESUMEN
Este artículo toma en cuenta las variables confianza y compromiso del cliente para lograr la tan deseada lealtad hacia a una marca. En este sentido, se propone validar un modelo teórico compuesto por tres pilares, es decir, la confianza, el compromiso y la lealtad de marca que será útil para las empresas que poseen un enfoque orientado hacia el valor de marca (brand equity). Este estudio ha sido uno de los pioneros en el contexto académico de Brasil en usar dichos pilares al analizar una marca con más de tres décadas de historia en el sector del entretenimiento musical brasileño, que es la del Festival Rock in Rio. Se alcanzaron 725 cuestionarios en línea por medio del Facebook Ads que fueron aplicados a los participantes del Rock in Rio en la edición del año 2017. Además, se establecieron, inicialmente, 3 hipótesis que se convirtieron en 5 hipótesis durante el proceso de análisis estadístico, de las que 4 de ellas fueron aceptadas. Según los resultados empíricos obtenidos, las relaciones entre los constructos confianza/expectativa de los servicios frente a compromiso, confianza/expectativa de los servicios frente a lealtad, confianza/recuperación de los servicios frente a lealtad y compromiso frente a lealtad presentaron resultados satisfactorios. Así que la utilización de dichos constructos en el modelo de prueba produjo un R2 de un $57,7 \%$ para el compromiso y un $83,1 \%$ para la lealtad, que son respectivamente positivos. Los hallazgos de este trabajo han sido tratados con análisis de contenido y procedimientos estadísticos basados en técnicas multivariantes.
\end{abstract}

Palabras clave: marca, confianza, compromiso, lealtad, Rock in Rio.

Clasificación JEL: M31; C16; C30; L83.

MSC2010: 62HXX; 62H15; 62H25. 


\title{
The relationship between trust, commitment and its effects on brand loyalty
}

\begin{abstract}
This article considers the customer's trust and commitment variables in attaining the much desired brand loyalty. In this sense, the present study proposed to validate a theoric model composed of three pillars - trust, commitment and brand loyalty, which would be useful for companies with a focus on brand equity. This study was one of the first in the Brazilian academic context to utilize the aforementioned pillars in analyzing a brand with more than three decades of history in the Brazilian music entertainment sector, this being the Rock in Rio Festival. By means of the Facebook Advertisements, 725 online questionnaires were responded by participants in the 2017 edition of the Rock in Rio. In addition, three hypotheses which initially were established became five 5 hypotheses during the statistical analysis process, 4 of which were supported. According to the empirical results obtained, the relationship between the trust/service expectation $\mathrm{X}$ commitment, trust/service expectation $\mathrm{X}$ loyalty, trust/service recovery $\mathrm{X}$ loyalty and commitment $\mathrm{X}$ loyalty constructs presented satisfactory results. Therefore, the use of the said constructs in the test model yielded for commitment an R2 of $57.7 \%$ and for loyalty an R2 of $83.1 \%$, each of which respectively is positive. The findings of this work were established on content analysis and statistical procedures based on multivariate techniques.
\end{abstract}

Keywords: brand, trust, commitment, loyalty, Rock in Rio.

JEL classification: M31; C16; C30; L83.

MSC2010: 62HXX; 62H15; 62H25.

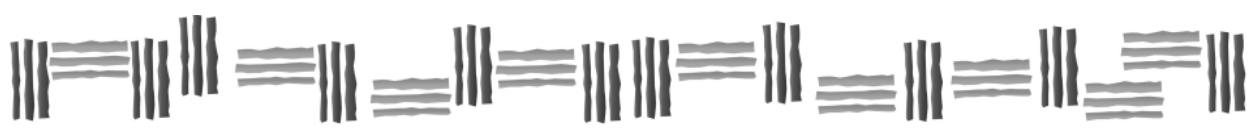




\section{Introducción.}

Hoy en día, la marca puede ser considerada uno de los temas más relevantes en los estudios de marketing. Ésta sigue siendo, para muchas empresas, el activo intangible más rentable y la base de la ventaja competitiva en un escenario cada vez más complejo y disputado. Asimismo, las empresas, en mayor medida, están cambiando sus esfuerzos mercadológicos a fin de generar vínculos a largo plazo con los consumidores, estas relaciones crean el éxito de larga duración para las empresas y sus marcas (Bastos et al., 2015). La creación y el mantenimiento de marcas fuertes es un desafío y, sobre todo, un diferencial competitivo para las empresas, siendo la confianza, el compromiso y la lealtad de marca enfoques fundamentales en los estudios de branding, es decir, de la gestión de marca (Ballester, 2004).

El objetivo de este trabajo es crear un modelo teórico con el propósito de verificar la relación entre la confianza de marca y el compromiso con la marca, además de la influencia y el impacto de esas variables en la lealtad de marca por el consumidor. Asimismo, se llevará a cabo la validez de las escalas utilizadas para medir la confianza, el compromiso y la lealtad de marca.

En términos teóricos, cabe señalar que, actualmente, la confianza y el compromiso están siendo estudiados por el marketing y que las relaciones resultantes entre éstos y la lealtad de marca de los consumidores es una contribución teórica relacionada con el vínculo entre los varios constructos relativos a las marcas cuyas relaciones son estudiadas en diversos modelos teóricos. Por lo tanto, los constructos confianza y compromiso se revelan como importantes antecedentes de la lealtad de marca en los estudios sobre marcas (Frederico, 2004; Ercis et al., 2012; Lee et al., 2015).

En términos prácticos, a partir de la confirmación de las hipótesis planteadas de esta investigación, los gestores podrán actuar en la administración del marketing con el intuito de aumentar la percepción de la confianza y del compromiso con la marca, generando así un mayor nivel de lealtad de marca de los clientes, justo lo que es importante para alcanzar los buenos resultados de las empresas.

\section{Revisión de la literatura.}

\subsection{Festival Rock in Rio.}

La marca objeto de análisis es la marca brasileña del festival Rock in Rio (RIR), realizado por la empresa Rock World S.A. Con más de treinta años de historia, RIR es considerado uno de los eventos de música y entretenimiento más grandes del mundo, que ha situado a Brasil en el circuito del show business internacional, teniendo como elemento clave la experiencia (Carneiro, 2011; Silva \& Tincani, 2013; Höpner, 2017; Rock in Rio, 2017). De las diecinueve ediciones hasta 2018, siete se realizaron en Brasil, ocho en Portugal, tres en España y una en los Estados Unidos.

El RlR ofrece variadas atracciones experienciales a su público objetivo, como, por ejemplo, en la edición del año 2017 en Río de Janeiro: "Yo me voy a casar en Rock in Rio", Mega Drop, Montaña Rusa, Tirolina, Rueda Gigante, Game XP, entre otras (Rock World, 2017).

A continuación, se presenta la Figura 1, con los datos del Festival Rock in Rio del año 1985 hasta 2016. 
Figura 1. Los números del gigante musical (1985-2016).

\begin{tabular}{|c|c|}
\hline \multirow[t]{9}{*}{ Rock in Rio } & 17 ediciones desde 1985 \\
\hline & 4 países \\
\hline & 101 días de magia desde 1985 \\
\hline & 1588 artistas seleccionados \\
\hline & 8,5 millones de personas en la platea \\
\hline & 3 millones de árboles donados a la Amazonia \\
\hline & 182 mil empleos generados \\
\hline & 11 millones de fans en línea \\
\hline & 500 millones de personas impactadas en 2015 \\
\hline
\end{tabular}

La propuesta de valor del RIR tiene la premisa de "proporcionar experiencias inolvidables a través de la música" (Rock World, 2016 citado por Höpner, 2017, p. 86, traducción de los autores).

El RIR se esfuerza constantemente en la construcción de experiencias de consumo, denominadas experiencia $360^{\circ}$, que no se dirigen al consumidor exclusivamente durante el festival, sino también antes y después del evento. Estas experiencias se desarrollan en función de las relaciones: (1) Consumidor-Rock in Rio que se realizan acciones de comunicación para interaccionar con el público objetivo para crear momentos, que son los que pueden ser recordados y contienen la historia de los participantes en el festival de diversas formas; (2) Rock in Rio Socios (partners) que se realizan acciones de comunicación para interaccionar con los socios y patrocinadores del RIR para que ellos puedan posibilitar experiencias a sus empleados y sus clientes (Höpner, 2017; Rock World, 2017).

\subsection{Confianza.}

Una de las principales líneas de estudio acerca de la confianza en la marca se basa en un tratamiento psicológico, cuyo fundamento reside en la expectativa del consumidor con relación a la confiabilidad ante una marca. La esencia de este enfoque consiste en la creencia de que los consumidores tienen en consideración las cualidades específicas que la marca posee y que la convierten en consistente, competitiva, honesta y responsable (Chaudhuri \& Holbrook, 2001; Ballester, 2004; Ballester \& Alemán, 2005).

Para Chaudhuri y Holbrook (2001), "la confianza de marca es la voluntad del consumidor de confiar sobre la capacidad de la marca para desempeñar su función declarada" (p. 82, traducción de los autores). En este mismo sentido, Sahin et al. (2011) afirman que la confianza en la marca puede ser entendida como la creencia del consumidor de que la empresa irá a cumplir aquello que se les ha prometido.

Ballester et al. (2003) y Bastos et al. (2015) identificaron que la confianza en la marca está significativamente relacionada con la lealtad de marca y proponen una escala válida y que refuerza la tesis de que la confianza en la marca es un constructo central en el desarrollo de las relaciones a largo plazo con los consumidores.

La confianza en la marca es uno de los factores más importantes que influye directamente en la lealtad de marca (Chaudhuri \& Holbrook, 2001; Sung \& Kim, 2008). Existen diversos estudios que explican los impactos significativos de la confianza en la lealtad de actitud y la lealtad de 
comportamiento (Matzler et al., 2006; Matzler et al., 2008; Anuwichhanont, 2011; Hanzaee \& Andervazh, 2012).

Lee et al. (2015) señalan que la confianza en la marca lleva a la lealtad o el compromiso de marca, porque la confianza crea relaciones de intercambio que son altamente valoradas. Una importante consecuencia de la confianza es una relación duradera. Estos investigadores que han examinado empíricamente la relación entre confianza y lealtad han encontrado que la confianza es un determinante clave de la lealtad de marca.

En el contexto empresarial, la confianza está relacionada con la habilidad y la capacidad atribuidas a una empresa para realizar algunas actividades y cumplir sus obligaciones y sus promesas con las partes interesadas (Bastos et al., 2015). Sin embargo, la confianza en la marca es algo que va más allá del resultado de las percepciones de los atributos del producto, puesto que es el resultado de las acciones de la empresa en nombre de la marca, asimismo, puede ser desarrollada y ser fruto de la experiencia con la marca (Ballester \& Alemán, 2001). Por lo tanto, la confianza en la marca está directamente relacionada con las experiencias vivenciadas por los consumidores con la marca, en que ésta se ha mostrado enfocada a la propuesta de valor promocionada y las expectativas de satisfacer las necesidades (Koetz et al., 2009; Bastos et al., 2015).

Breitenbach (2016) sostiene, a través de sus estudios, la confirmación de que la confianza en la marca impacta positivamente en el compromiso, en consonancia con los modelos orientativos y también con la literatura contemporánea acerca de dicho tema (Guo et al., 2015; Ponder et al., 2016). La confianza en la marca y el compromiso ejercen papeles complementares en la relación entre marcas y consumidores, además deben ser objeto de estrategias específicas por parte de las empresas, según señala Breitenbach (2016).

A partir de lo anterior, se pueden establecer las siguientes hipótesis:

\section{H1: La confianza tiene un efecto significativamente positivo sobre el compromiso. H2: La confianza tiene un efecto significativamente positivo sobre la lealtad.}

\subsection{Compromiso.}

El compromiso ha recibido una gran atención tanto a lo largo de toda la literatura del marketing como en los últimos trabajos de investigación que han abordado, específicamente, el compromiso entre consumidor y marcas (Fullerton, 2003, 2014; Evanschitzky et al., 2006; Kim et al., 2008; Kemp \& Bui, 2011; Ercis et al., 2012; Mathew et al., 2012; Kazemi et al., 2013; Sallam, 2015).

Dicho concepto aparece, también, reconocido como un importante ingrediente esencial para el éxito de las relaciones de forma prolongada entre empresas y clientes (Morgan \& Hunt, 1994; Palmatier et al., 2006; Palmatier, 2008; Palmatier et al., 2013; Guede, 2014; Breitenbach, 2016).

El compromiso puede ser traducido como un deseo duradero del consumidor en mantener una relación con una marca (Moorman et al., 1992) y estaría caracterizado por apegos económicos, emocionales y psicológicos de consumidores y marcas (Evanschitzky et al., 2006). Desde un punto de vista experiencial, el compromiso puede ser considerado como una segunda fuente de experiencia más allá de la fuente hedónica de la experiencia, que es resultante de una fuerza motivacional para hacer o no que algo acontezca (Higgins, 2006; Doorn et al., 2010). Para Guede (2014) el compromiso puede ser entendido como el nivel del estado de ánimo de una persona que resulta de comparar el rendimiento percibido de un producto o servicio con sus experiencias.

Considerando el contexto de las marcas, Ercis et al. (2012), Kazemi et al. (2013) y Sallam (2015) defienden que el compromiso puede dividirse en dos componentes: compromiso emocional (o afectivo) y compromiso de continuidad. Existe una relación significativa entre el compromiso emocional (o 
afectivo) y la marca; esto se refiere al poderoso sentimiento personal y emocional basado en la identificación y los valores compartidos de la marca, es decir, se refiere a la profunda pertenencia hacia ésta. En el compromiso de continuidad, el cliente podrá cambiar de marca según su estado psicológico temporal, puesto que no existe un fuerte sentimiento hacia ella.

Evanschitzky et al. (2006) subrayan en sus investigaciones que el compromiso afectivo, en comparación con el compromiso de continuidad, según ya fue abordado por otro autor (Fullerton, 2003), presenta una mayor influencia en la lealtad. El impacto relativamente más fuerte del compromiso afectivo, comparado con el compromiso de continuidad, es consistente e impulsa el comportamiento de lealtad. Este hallazgo tiene importantes implicaciones para la lealtad del cliente que la reconoce como una construcción multidimensional.

De acuerdo con los resultados obtenidos en el artículo de Ercis et al. (2012), el papel de la satisfacción y el compromiso de continuidad en la lealtad es menor, mientras que la confianza y el compromiso afectivo tienen un efecto importante en la creación de la lealtad del consumidor. Por lo que estos autores sugieren que las empresas den más importancia a la creación de confianza y compromiso afectivo para lograr lealtad hacia sus marcas, porque el compromiso de continuidad no demuestra que los consumidores sean totalmente leales. Para tener éxito, las empresas deben ofrecer diversas y válidas razones para que sus clientes continúen comprando sus productos y sus servicios al entrar en el mundo de los consumidores y, de esta forma, alcanzar la completa lealtad (Ercis et al., 2012).

Por último, Mathew et al. (2012) añaden el papel del compromiso de marca sobre las intenciones de lealtad en la formación del valor de marca (brand equity).

A partir de lo anterior, se puede establecer la siguiente hipótesis:

\section{H3: El compromiso tiene un efecto significativamente positivo sobre la lealtad.}

\subsection{Lealtad.}

La variable lealtad se asume como un esfuerzo de la empresa para mantener la conexión con el cliente, siendo así de vital importancia su creación y su sustentación diaria, debido a la fuerte competencia del contexto actual. La lealtad tiene, de esta manera, una importancia mayor en la estrategia empresarial, lo que obliga a una mayor comprensión de su dimensión (Rodrigues, 2015).

Ballester (2004) subraya que:

mientras que para unos investigadores la lealtad se manifiesta como la recompra persistente de una marca a lo largo del tiempo, para otros toma la forma de un compromiso psicológico del consumidor con la marca que se traduce en una actitud positiva y en una intención de recompra. (p.17)

Colmenares y Saavedra (2007) sostienen que la lealtad se orienta en tres enfoques: comportamental, actitudinal y actitudinal-comportamental. La lealtad comportamental ocurre en la repetición en el momento de la compra de un mismo producto, una marca o un proveedor. La lealtad actitudinal sucede en el compromiso psicológico del consumidor, según los sentimientos y los afectos positivos de una empresa, una marca o un producto de acuerdo con la experiencia en relación con sus necesidades. Y, por último, la lealtad actitudinal-comportamental, que se produce cuando la lealtad no concierne solo al comportamiento de recompra o al compromiso, sino a ambos, como un compromiso psicológico del consumidor con la marca.

Según el estudio de Henning-Thurau et al. (2002), los constructos: confianza y compromiso están relacionados directamente con la lealtad. Estos autores señalan que la lealtad resulta mejor explicada cuando se considera el efecto mediador de la confianza y el compromiso, que cuando se proponía un efecto aislado de estos constructos sobre la lealtad. 


\section{Metodología.}

Con el fin de confirmar las hipótesis creadas y presentadas previamente para este proyecto de investigación, los autores de este artículo han aplicado la siguiente metodología científica: enfoque cuantitativo por medio de una encuesta en línea.

\subsection{Enfoque cuantitativo.}

El enfoque cuantitativo, según Sampieri et al. (2006), "usa la recolección de datos para probar hipótesis, con base en la medición numérica y el análisis estadístico, para establecer patrones de comportamiento y probar teorías" (p.6, traducción de los autores).

La investigación del tipo descriptiva se basa en una recolección de datos estructurada y sometida a la respuesta del número (muestra) representativo de la población de estudio (Hair et al., 2010; Malhotra et al., 2012), consistiendo en las fases de recolectar datos, tabular los datos, calcular la frecuencia para las variables investigadas e interacciones entre sí, además de los respectivos análisis y las interpretaciones del mismo objeto (Malhotra et al., 2012). Los estudios descriptivos, normalmente, se estructuran en función de hipótesis o preguntas investigativas, previamente estructuradas e integrantes del modelo teórico (Churchill, 1999).

\subsection{Encuesta en línea.}

En este artículo, considerando el objetivo y las hipótesis planteadas se ha aplicado la técnica de la encuesta en línea, ya que, para obtener la información primaria, se ha necesitado realizar a los individuos una serie de preguntas sobre sus comportamientos, sus intenciones, sus actitudes y sus conocimientos sobre la marca investigada.

\subsection{Diseño de la encuesta en línea.}

Con el fin de confirmar las hipótesis que se ven al comienzo de este artículo, se ha llevado a cabo una técnica de encuesta en línea a los participantes del festival RIR en la edición del año 2017 en la ciudad de Río de Janeiro (Brasil). El cuestionario aplicado se ha estructurado en cuatro bloques para recopilar información, actitudes y opiniones sobre la experiencia de marca de RIR.

A continuación, se presenta la Tabla 1 con los constructos, los códigos, los ítems y los autores que han servido de referencia para desarrollar los bloques 1, 2 y 3 del cuestionario. Dicho cuestionario fue elaborado en portugués, porque, al celebrarse en Brasil, la mayoría de los asistentes tenían como lengua materna este idioma. Éste fue probado el día 15 de septiembre de 2017, en el primer día del festival RIR en la Ciudad del Rock, con una muestra de 35 personas de diferentes edades, géneros y nivel de estudios, con el fin de garantizar la adecuada comprensión de las preguntas; no ha sido necesario adecuar el cuestionario y tampoco esos cuestionarios probados fueron incorporados en la muestra. Tomó más de 8 minutos completar un cuestionario en línea y con un índice de respuesta del $42 \%$ al considerar la herramienta utilizada. Se han utilizado dos acciones para recolección de datos: contacto directo y Facebook Ads. Fueron 1.018 cuestionarios en línea contestados, de los cuales 725 cuestionarios han sido válidos para la investigación empírica. Para medir las variables de las preguntas, se aplicaron escalas intervalo de diez puntos para determinar la sensibilidad de estas variables. Los cuestionarios en línea han sido facilitados por el enlace disponible en la campaña publicitada en el Facebook Ads y estuvo disponible durante 20 días en el mes de diciembre de 2017. Los cuestionarios contestados por la acción de contacto directo estuvieron disponibles en los meses de octubre, noviembre y diciembre del año 2017, con un insignificante índice de respuesta. Por lo tanto, la población total consistió en todos los participantes del Festival RIR que asistieron a la edición del año 2017. Los autores han tomado una muestra de 725 encuestados (muestreo no probabilístico). Finalmente, los datos primarios obtenidos se analizaron con los paquetes estadísticos para ciencias sociales (IBM SPSS V.20 y AMOS V.21) para análisis multivariante. 
Tabla 1. Constructos, códigos, ítems y autores consultados.

\begin{tabular}{|c|c|c|c|}
\hline Constructo & Código & Ítem & Referencia bibliográfica \\
\hline \multirow{8}{*}{ Confianza } & CONF1 & El Rock in Rio cumple mis expectativas. & \multirow{8}{*}{ Ballester et al. (2003) } \\
\hline & CONF2 & El Rock in Rio nunca me decepciona. & \\
\hline & CONF3 & Yo siento confianza en el nombre Rock in Rio. & \\
\hline & CONF4 & El Rock in Rio es una garantía de satisfacción. & \\
\hline & CONF5 & $\begin{array}{l}\text { El Rock in Rio es honesto y sincero cuando se refiere } \\
\text { a mis intereses. }\end{array}$ & \\
\hline & CONF6 & $\begin{array}{l}\text { Yo podría contar con el Rock in Rio para resolver mis } \\
\text { problemas relacionados con los servicios prestados. }\end{array}$ & \\
\hline & CONF7 & $\begin{array}{l}\text { El Rock in Rio haría cualquier esfuerzo para } \\
\text { satisfacerme como cliente. }\end{array}$ & \\
\hline & CONF8 & $\begin{array}{l}\text { El Rock in Rio me compensaría de alguna manera por } \\
\text { cualquier problema con sus servicios. }\end{array}$ & \\
\hline \multirow{8}{*}{ Compromiso } & COMP1 & $\begin{array}{l}\text { Sería muy difícil cambiar el Rock in Rio por otro } \\
\text { festival, aunque yo quisiera. }\end{array}$ & \multirow{8}{*}{ Ercis et al. (2012) } \\
\hline & COMP2 & $\begin{array}{l}\text { Sería una pérdida para mí si yo cambiase el Rock in } \\
\text { Rio por otro festival de música. }\end{array}$ & \\
\hline & COMP3 & Me siento fuertemente conectado con el Rock in Rio. & \\
\hline & COMP4 & $\begin{array}{l}\text { Compensa en términos económicos ser cliente del } \\
\text { Rock in Rio. }\end{array}$ & \\
\hline & COMP5 & $\begin{array}{l}\text { Sería malo económicamente para mí si dejara de ser } \\
\text { cliente del Rock in Rio. }\end{array}$ & \\
\hline & COMP6 & $\begin{array}{l}\text { El Rock in Rio tiene una ubicación mejor (está mejor } \\
\text { situado) en comparación con otros festivales. }\end{array}$ & \\
\hline & COMP7 & $\begin{array}{l}\text { El Rock in Rio se interesa en cómo participo en los } \\
\text { conciertos del festival. }\end{array}$ & \\
\hline & COMP8 & $\begin{array}{l}\text { Siempre doy retorno al Rock in Rio sobre mis } \\
\text { percepciones del festival. }\end{array}$ & \\
\hline \multirow{5}{*}{ Lealtad } & LEA1 & Me considero leal al Rock in Rio. & \multirow{5}{*}{ Oliver (1999) } \\
\hline & LEA2 & $\begin{array}{l}\text { Incluso si hay otras opciones, el Rock in Rio es mi } \\
\text { primera opción de conciertos y festivales de música. }\end{array}$ & \\
\hline & LEA3 & Creo que vale la pena seguir yendo al Rock in Rio. & \\
\hline & LEA4 & $\begin{array}{l}\text { Si mis condiciones económicas me lo permiten, } \\
\text { prefiero ir al Rock in Rio. }\end{array}$ & \\
\hline & LEA5 & Yo iré al Rock in Río de nuevo. & \\
\hline
\end{tabular}

Fuente: Elaboración propia.

\section{Análisis de los datos.}

\subsection{Detección de los outliers multivariantes: cálculo de la distancia de Mahalanobis $\left(\mathrm{D}^{2}\right)$.}

Los outliers multivariantes deben ser identificados y analizados con objetivo de eliminarlos o no, pues pueden incidir sobre los resultados finales del análisis estadístico. Así, de esta manera, fueron calculados los valores de la distancia de Mahalanobis $\left(\mathrm{D}^{2}\right)$ para todos los elementos de la muestra y todos aquellos que presentaron un valor de $\mathrm{D}^{2}$ arriba del valor de la prueba Chi-cuadrado $\left(X^{2}\right)$ con el número de grados de libertad correspondientes al número de las variables y con significación de 0,001 han sido considerados outliers multivariantes (Hair et al., 2009). Como resultado final, fueron identificados y retirados 155 outliers multivariantes. Por consiguiente, la muestra final se ha quedado con 725 elementos. 


\subsection{Prueba de normalidad multivariante Kolmogorov-Smirnov (K-S).}

Según Malhotra (2010), la prueba de Kolmogorov-Smirnov (K-S) consiste en el contraste de la función cumulativa de una variable con una distribución específica. Para que la distribución sea considerada normal, el valor de significación (sig.) debe ser mayor de 0,05 y que la distribución sea considerada anormal (o no normal), el valor de significación (sig.) debe ser inferior a 0,05 .

Los resultados obtenidos indican que la muestra de este estudio no sigue una distribución normal para todos los ítems investigados.

\subsection{Distribución de la frecuencia: características de la muestra.}

La muestra para el estudio empírico se ha realizado en el ámbito nacional de Brasil, en 196 ciudades, de las cuales se obtuvieron 725 encuestados, y posee las principales características demográficas: brasileños, sexo femenino, solteras, 18 años hasta 34 años, con una educación superior completa, ingresos desde cinco hasta diez sueldos mínimos mensuales (R\$ 937,00 ha sido el sueldo mínimo en Brasil en la fecha de recolección de datos (diciembre 2017), 937 reales $=201,50$ euros $/$ Tasa de cambio $4,65 €$ en 01/10/18) y viven en la región sudeste de Brasil, que es la misma región de origen del festival RIR.

La muestra utilizada, por tanto, está compuesta por miembros de la clase media de Brasil, lo que está en consonancia con el público objetivo del festival RIR y sus políticas de precio.

\subsection{Análisis descriptivo: cálculo de la media.}

El análisis descriptivo de los datos fue realizado a partir del cálculo de la media del $n$ muestral, siendo mínimo 0 y máximo 10, de cada variable de los constructos que componen el modelo de prueba.

El constructo confianza, de acuerdo con la Tabla 2, presentó un promedio de 8,67. El ítem CONF8 exhibe el menor promedio de este constructo de 8,09. Mientras que el ítem CONF3 muestra el mayor promedio de 9,32. Un resultado predecible, dado que el RIR tiene más de tres décadas de historia y está entre los mayores eventos de música y entretenimiento del mundo (Carneiro, 2011; Silva \& Tincani, 2013; Höpner, 2017; Rock in Rio, 2017). A los clientes que desistieron de asistir el primer día de la edición de 2017 del RIR porque Lady Gaga canceló su participación, fueron resarcidos con la devolución del pago de las entradas/pulseras.

Tabla 2. Análisis descriptivo del constructo: confianza.

\begin{tabular}{|c|c|c|c|c|}
\hline Constructo / Ítem & $n$ & Mínimo & Máximo & Media \\
\hline Confianza & 725 & n.d $d^{a}$ & n.d & 8,67 \\
\hline CONF1 - El Rock in Rio cumple mis expectativas. & 725 & 4 & 10 & 9,25 \\
\hline CONF2 - El Rock in Rio nunca me decepciona. & 725 & 1 & 10 & 8,48 \\
\hline CONF3 - Yo siento confianza en el nombre Rock in Rio. & 725 & 4 & 10 & 9,32 \\
\hline CONF4 - El Rock in Rio es una garantía de satisfacción. & 725 & 3 & 10 & 9,04 \\
\hline $\begin{array}{l}\text { CONF5 - El Rock in Rio es honesto y sincero cuando se refiere a mis } \\
\text { intereses. }\end{array}$ & 725 & 3 & 10 & 8,79 \\
\hline $\begin{array}{l}\text { CONF6 - Yo podría contar con el Rock in Rio para resolver mis problemas } \\
\text { relacionados con los servicios prestados. }\end{array}$ & 725 & 0 & 10 & 8,14 \\
\hline $\begin{array}{l}\text { CONF7 - El Rock in Rio haría cualquier esfuerzo para satisfacerme como } \\
\text { cliente. }\end{array}$ & 725 & 2 & 10 & 8,26 \\
\hline $\begin{array}{l}\text { CONF8 - El Rock in Rio me compensaría de alguna manera por cualquier } \\
\text { problema con sus servicios. }\end{array}$ & 725 & 0 & 10 & 8,09 \\
\hline
\end{tabular}

${ }^{\mathrm{a}}$ No disponible

Fuente: Elaboración propia. 
De esa manera, dicho constructo también presenta un alto promedio, de lo que se puede inferir que la marca del festival RIR posee una relación de confianza con sus clientes que ha sido construida desde la primera edición en 1985.

Tabla 3. Análisis descriptivo del constructo: compromiso.

\begin{tabular}{ccccc}
\hline Constructo / Ítem & $n$ & Mínimo & Máximo & Media \\
\hline Compromiso & 725 & n.d & n.d & 7,86 \\
COMP1 - Sería muy difícil cambiar el Rock in Rio por otro festival, aunque & 725 & 0 & 10 & 8,47 \\
$\begin{array}{l}\text { yo quisiera. } \\
\text { COMP2 - Sería una pérdida para mí si yo cambiase el Rock in Rio por otro } \\
\text { festival de música. }\end{array}$ & 725 & 0 & 10 & 7,68 \\
$\begin{array}{l}\text { COMP3 - Me siento fuertemente conectado con el Rock in Rio. } \\
\text { COMP4 - Compensa en términos económicos ser cliente del Rock in Rio. }\end{array}$ & 725 & 0 & 10 & 8,86 \\
COMP5 - Sería malo económicamente para mí si dejara de ser cliente del & 725 & 0 & 10 & 8,08 \\
$\begin{array}{l}\text { Rock in Rio. } \\
\text { COMP6 - El Rock in Rio tiene una ubicación mejor (está mejor situado) en } \\
\text { comparación con otros festivales. }\end{array}$ & 725 & 0 & 10 & 5,63 \\
$\begin{array}{c}\text { COMP7 - El Rock in Rio se interesa en cómo participo en los conciertos } \\
\text { del festival. }\end{array}$ & 725 & 1 & 10 & 8,58 \\
COMP8 - Siempre doy retorno al Rock in Rio sobre mis percepciones del & 725 & 0 & 10 & 7,31 \\
\hline festival.
\end{tabular}

Fuente: Elaboración propia.

En la Tabla 3, se puede visualizar que el constructo compromiso presentó el menor promedio de 7,86 , en comparación con los demás constructos analizados. El ítem COMP5 exhibe el menor promedio de este constructo de 5,63. Mientras que el ítem COMP3 mostró el mayor promedio de 8,86. Este resultado es predecible debido al valor de las entradas/pulseras, en la edición de 2017, tuvieron un coste unitario de $\mathrm{R} \$ 455,00 /$ día (455 reales $=97,85 € /$ Tasa de cambio 4,65€ en 01/10/18), lo que se considera caro en función del sueldo mínimo de los brasileños que es de R $\$ 954,00$ (954 reales $=205,16$ $€ /$ Tasa de cambio 4,65€ en 01/10/18) en 2018 (IBGE, 2018). Así que, en contraste con la COMP5, sería bueno económicamente dejar de ser cliente del RIR desde el punto de vista del ahorro, pero la conexión de los clientes con la marca es demasiado fuerte y ésta no se lo permite. Aunque el constructo compromiso muestre el menor promedio en comparación con los demás, todavía se considera alto y significativo. Así que el festival RIR busca, continuamente, conectarse con sus clientes con el objetivo de ofrecer la experiencia $360^{\circ}$.

Tabla 4. Análisis descriptivo del constructo: lealtad.

\begin{tabular}{lcccc}
\hline \multicolumn{1}{c}{ Constructo / Ítem } & $\boldsymbol{n}$ & Mínimo & Máximo & Media \\
\hline \multicolumn{1}{c}{ Lealtad } & 725 & n.d da $^{\mathrm{a}}$ & n.d & 9,28 \\
LEA1 - Me considero leal al Rock in Rio. & 725 & 2 & 10 & 8,82 \\
LEA2 - Incluso si hay otras opciones, el Rock in Rio es mi primera opción & 725 & 2 & 10 & 8,96 \\
$\quad$ de conciertos y festivales de música. & 725 & 3 & 10 & 9,57 \\
LEA3 - Creo que vale la pena seguir yendo al Rock in Rio. & 725 & 2 & 10 & 9,43 \\
LEA4 - Si mis condiciones económicas me lo permiten, prefiero ir al & 725 & & \\
$\quad$ Rock in Rio. & 725 & 2 & 10 & 9,61 \\
\hline LEA5 - Yo iré al Rock in Rio de nuevo. & & & &
\end{tabular}

${ }^{a}$ No disponible

Fuente: Elaboración propia.

De acuerdo con el constructo lealtad, como se puede visualizar en la Tabla 4, el promedio presentado ha sido el más alto de los demás constructos analizados de 9,28. El ítem LEA1 exhibió el 
menor promedio de este constructo de 8,82. Mientras que el ítem LEA5 mostró el promedio de 9,61, considerado el más alto de todos los ítems analizados. Este resultado, como los anteriores, era previsible, puesto que en Brasil se ofrecen otros festivales internacionales similares para el mismo público objetivo del RIR, como el Lollapalooza, por ejemplo. No obstante, el RIR cuenta con una alta fidelización en su Rock in Rio Club que ofrece varios beneficios a los clientes asociados, desde la compra anticipada de las entradas/pulseras hasta descuentos en los más de 700 productos comercializados en la tienda oficial del RIR, ubicada en la Ciudad del Rock. Cabe destacar que, en 2015, el Rock in Rio Card estuvo agotado en apenas 50 minutos de adhesión en línea. Así que es posible inferir que el festival RIR posee un público objetivo con alto índice de lealtad con su marca.

\subsection{Análisis factorial exploratorio (AFE).}

Según Hair et al. (2009), el análisis factorial exploratorio (AFE), en el enfoque estadístico utilizado, permite analizar las interrelaciones entre un gran número de variables y explicar esas variables en términos de sus dimensiones específicas comunes. Para llevar a cabo el AFE es necesario atender a estos tres presupuestos: medida de adecuación muestral de Kaiser-Meyer-Olkin (KMO), prueba de esfericidad de Bartlett y matriz de correlación, teniendo en cuenta que todos los ítems que forman el constructo deben presentar un alto nivel de correlación entre sí.

Tabla 5. Medida de adecuación muestral de Kaiser-Meyer-Olkin (KMO) y Prueba de esfericidad de Bartlett.

\begin{tabular}{cccccc}
\hline $\begin{array}{c}\text { Constructos definidos } \\
\text { por el modelo }\end{array}$ & $\begin{array}{c}\text { Medida de } \\
\text { Adecuación } \\
\text { muestral de } \\
\text { KMO }\end{array}$ & Prueba de esfericidad de Bartlett & $\begin{array}{c}\text { Número de } \\
\text { correlaciones } \\
\text { entre los } \\
\text { ítems del } \\
\text { constructo }\end{array}$ & $\begin{array}{c}\text { Número de } \\
\text { correlaciones } \\
\text { estadísticas } \\
\text { con } \\
\text { significación }\end{array}$ \\
\hline Confianza & 0,906 & 3519,045 & 0,000 & 28 & 28 \\
Compromiso & 0,871 & 2110,754 & 0,000 & 28 & 28 \\
Lealtad & 0,834 & 1993,325 & 0,000 & 10 & 10 \\
\hline
\end{tabular}

Fuente: Elaboración propia.

Según la Tabla 5, se puede verificar que todos los constructos presentaron el KMO mayor que 0,50 , lo que cumple favorablemente con el supuesto. Asimismo, se subraya que dichos constructos mostraron el KMO en nivel de excelencia conforme Hair et al. (2009) en su jerarquía de análisis de KMO.

En la Tabla 6, se puede ver que el constructo confianza presentó dos dimensiones (componente 1 y componente 2) a partir de los ítems utilizados con una varianza explicada de un $74,244 \%$ para los dos componentes. En función de los resultados obtenidos, el ítem CONF5 mostró una elevada carga de valor en los dos componentes, así que éste será retirado y no será tenido en cuenta en el próximo apartado. Asimismo, los resultados obtenidos fueron satisfactorios porque todos los ítems de dicho constructo presentaron valores de componente superiores a 0,675 y valores de comunalidad superiores a 0,678 .

Por consiguiente, se forman dos factores para el constructo confianza. El primer factor se llama "confianza/expectativa de los servicios" y el segundo factor, "confianza/recuperación de los servicios". Esos nombres fueron elegidos a partir del análisis de los ítems que componen cada uno de los constructos. 
Tabla 6. Análisis factorial del constructo: confianza.

\begin{tabular}{|c|c|c|c|}
\hline Descripción del ítem & Componente 1 & Componente 2 & Comunalidad \\
\hline CONF1 - El Rock in Rio cumple mis expectativas. & 0,800 & & 0,702 \\
\hline CONF2 - El Rock in Rio nunca me decepciona. & 0,814 & & 0,705 \\
\hline CONF3 - Yo siento confianza en el nombre Rock in Rio. & 0,794 & & 0,719 \\
\hline CONF4 - El Rock in Rio es una garantía de satisfacción. & 0,850 & & 0,802 \\
\hline $\begin{array}{l}\text { CONF5 - El Rock in Rio es honesto y sincero cuando se } \\
\text { refiere a mis intereses. }\end{array}$ & $\underline{0,675}$ & $\underline{0,472}$ & 0,678 \\
\hline $\begin{array}{l}\text { CONF6 - Yo podría contar con el Rock in Rio para resolver } \\
\text { mis problemas relacionados con los servicios } \\
\text { prestados. }\end{array}$ & & 0,827 & 0,755 \\
\hline $\begin{array}{l}\text { CONF7 - El Rock in Rio haría cualquier esfuerzo para } \\
\text { satisfacerme como cliente. }\end{array}$ & & 0,839 & 0,807 \\
\hline $\begin{array}{l}\text { CONF8 - El Rock in Rio me compensaría de alguna manera } \\
\text { por cualquier problema con sus servicios. }\end{array}$ & & 0,843 & 0,772 \\
\hline $\begin{array}{l}\text { Varianza explicada por el componente principal }=41,821 \% \\
\text { Varianza explicada por los dos componentes }=74,244 \%\end{array}$ & & & \\
\hline
\end{tabular}

Fuente: Elaboración propia.

En la Tabla 7, se muestra que el constructo compromiso presentó una varianza explicada de un $49,329 \%$. Los resultados obtenidos son satisfactorios porque todos los ítems de dicho constructo exhibieron valores de componente superiores a 0,612 y valores de comunalidad superiores a 0,375. Según Moura (2010), en algunas situaciones expuestas en su tesis doctoral, los valores de comunalidad pueden estar entre 0,300 y 0,500 .

Tabla 7. Análisis factorial del constructo: compromiso.

\begin{tabular}{lcc}
\hline \multicolumn{1}{c}{ Descripción del ítem } & Componente & Comunalidad \\
\hline COMP1 - Sería muy difícil cambiar el Rock in Rio por otro festival, aunque & 0,764 & 0,583 \\
$\begin{array}{c}\text { yo quisiera. } \\
\text { COMP2 - Sería una pérdida para mí si yo cambiase el Rock in Rio por otro } \\
\quad \text { festival de música. }\end{array}$ & 0,808 & 0,654 \\
COMP3 - Me siento fuertemente conectado con el Rock in Rio. & 0,793 & 0,629 \\
COMP4 - Compensa en términos económicos ser cliente del Rock in Rio. & 0,708 & 0,501 \\
COMP5 - Sería malo económicamente para mí si dejara de ser cliente del & 0,612 & 0,375 \\
$\begin{array}{c}\text { Rock in Rio. } \\
\text { COMP6 - El Rock in Rio tiene una ubicación mejor (está mejor situado) }\end{array}$ & 0,616 & 0,379 \\
$\begin{array}{c}\text { en comparación con otros festivales. } \\
\text { COMP7 - El Rock in Rio se interesa en cómo participo en los conciertos } \\
\quad \text { del festival. }\end{array}$ & 0,669 & 0,448 \\
COMP8 - Siempre doy retorno al Rock in Rio sobre mis percepciones del \\
$\begin{array}{c}\text { festival. } \\
\text { Varianza explicada por el componente }=49,329 \%\end{array}$ & 0,615 & 0,378 \\
\hline
\end{tabular}

\section{Fuente: Elaboración propia.}

Como se puede visualizar en la Tabla 8 , el constructo lealtad presentó una varianza explicada de un $68,460 \%$. Los resultados obtenidos son satisfactorios porque todos los ítems de dicho constructo revelan valores de componente superiores a 0,783 y valores de comunalidad superiores a 0,612 . 
Tabla 8. Análisis factorial del constructo: lealtad.

\begin{tabular}{lcc}
\hline \multicolumn{1}{c}{ Descripción del ítem } & Componente & Comunalidad \\
\hline LEA1 - Me considero leal al Rock in Rio. & 0,783 & 0,612 \\
LEA2 - Incluso si hay otras opciones, el Rock in Rio es mi primera opción de & 0,836 & 0,699 \\
$\quad$ conciertos y festivales de música. & 0,886 & 0,785 \\
LEA3 - Creo que vale la pena seguir yendo al Rock in Rio. & 0,828 & 0,685 \\
LEA4 - Si mis condiciones económicas me lo permiten, prefiero ir al Rock in & 0,801 & 0,641 \\
$\quad$ Rio. & & \\
LEA5 - Yo iré al Rock in Rio de nuevo. & & \\
Varianza explicada por el componente $=68,460 \%$ &
\end{tabular}

Fuente: Elaboración propia.

\subsection{Análisis de fiabilidad de las escalas: cálculo del Alfa de Cronbach.}

Para Malhotra (2010) la fiabilidad de la consistencia interna del instrumento debe ser calculada por el coeficiente Alfa de Cronbach. En la Tabla 9, se puede visualizar que todos los constructos investigados alcanzaron valores de Alfa de Cronbach entre 0,700 y 0,900, lo que es considerado ideal según Malhotra (2010).

Tabla 9. Valores de Alfa de Cronbach de los constructos investigados.

\begin{tabular}{cc}
\hline Constructo & Alfa de Cronbach \\
\hline Confianza/expectativa de los servicios & 0,869 \\
Confianza/recuperación de los servicios & 0,858 \\
Compromiso & 0,833 \\
Lealtad & 0,867 \\
\hline
\end{tabular}

Fuente: Elaboración propia.

\subsection{Validez convergente por medio del cálculo de la Varianza media extraída (AVE) y la Fiabilidad compuesta (FC).}

La validez convergente es la medida de validez del constructo que mide el grado en que la escala se correlaciona positivamente con otras medidas del mismo constructo. Esta prueba tiene como objetivo verificar hasta qué punto la escala se relaciona positivamente con otras medidas del mismo constructo. Altas cargas factoriales indican que las variables analizadas son percibidas de forma semejante por los encuestados. La validez convergente evidencia la fuerte correlación entre las medidas de un mismo constructo (Malhotra, 2010; Hair et al., 2009).

Como se puede verificar, en la Tabla 10, los 4 constructos presentaron valores superiores de 0,500 en la AVE. En relación con la FC, los 4 constructos presentaron valores superiores a 0,700 , lo que es considerado satisfactorio según los autores mencionados.

En relación con el constructo compromiso, se puede notar que presentó un valor bajo según el valor recomendable por dichos autores, en el primer cálculo de la AVE. Así que los ítems de ese constructo que exhibieron las menores cargas estandarizadas también fueron retirados (COMP5, COMP6, COMP7 y COMP8) para una mejor adecuación de la validez convergente. 
Tabla 10. Validez convergente.

\begin{tabular}{ccc}
\hline Constructos & $\begin{array}{c}\text { (AVE) } \\
\text { Varianza Media } \\
\text { Extraída }\end{array}$ & $\begin{array}{c}\text { (FC) } \\
\text { Fiabilidad Compuesta }\end{array}$ \\
\hline Confianza/expectativa de los servicios & 0,660 & 0,886 \\
Confianza/recuperación de los servicios & 0,676 & 0,862 \\
Lealtad & 0,652 & 0,903 \\
Compromiso & 0,581 & 0,845 \\
\hline
\end{tabular}

Fuente: Elaboración propia.

Por último, se subraya que, en los análisis siguientes de validez discriminante, la validez nomológica y la prueba de hipótesis del constructo compromiso está formado apenas por sus cuatro primeros ítems (COMP1, COMP2, COMP3 y COMP4).

\subsection{Validez discriminante. Correlación entre los constructos y cálculo de la raíz cuadrada de la} AVE.

Para Hair et al. (2009) la validez discriminante está presente cuando se utilizan instrumentos para medir diferentes constructos y sus puntuaciones están débilmente correlacionadas, así que existe validez discriminante cuando el valor de la raíz cuadrada de la AVE es superior a la correlación entre los constructos.

En la Tabla 11, se puede notar que, de las seis combinaciones posibles, solamente en un caso el valor de la correlación entre los constructos fue mayor que el valor de la raíz cuadrada de la AVE: compromiso frente a lealtad, así que este caso no presentó validez discriminante. Las cinco combinaciones restantes en la matriz mostraron correlaciones inferiores a la raíz cuadrada de la AVE, lo que se confirma la existencia de validez discriminante en la mayoría de los casos analizados. Por consiguiente, esos resultados serán considerados en el próximo apartado de validez nomológica.

Tabla 11. Validez discriminante.

\begin{tabular}{|c|c|c|c|c|}
\hline Constructos & Compromiso & $\begin{array}{l}\text { Confianza/expectativa } \\
\text { de los servicios }\end{array}$ & $\begin{array}{c}\text { Confianza/recuperación } \\
\text { de los servicios }\end{array}$ & Lealtad \\
\hline Compromiso & 0,7620 & & & \\
\hline $\begin{array}{l}\text { Confianza/expectativa } \\
\text { de los servicios }\end{array}$ & 0,7069 & 0,8123 & & \\
\hline $\begin{array}{l}\text { Confianza/recuperación } \\
\text { de los servicios }\end{array}$ & 0,5760 & 0,6759 & 0,8223 & \\
\hline Lealtad & $\underline{0,9014}$ & 0,7687 & 0,5729 & 0,8075 \\
\hline
\end{tabular}

Fuente: Elaboración propia.

\subsection{Validez nomológica y prueba de hipótesis. Modelos de ecuaciones estructurales (SEM).}

Hair et al. (2009) afirman que la validez nomológica verifica si las relaciones y correlaciones entre los constructos investigados poseen significación y son coincidentes con las teorías y los conceptos presentados. Es un tipo de validez que determina la relación existente entre los constructos teóricos presentes en la teoría o los modelos teóricos que son estudiados. El proceso de análisis de validez nomológica empieza con la aplicación de la técnica de Modelos de ecuaciones estructurales (SEM = Structural Equation Modeling), en la cual se utiliza el modelado de ecuaciones estructurales para 
confirmar y explicar los modelos conceptuales que tratan las actitudes, las percepciones y otros factores que determinan el comportamiento de las variables.

En la Figura 2, se presentan los resultados de la SEM del modelo de prueba con la representación de las cargas estandarizadas en las flechas de los constructos.

Figura 2. Resultados de la SEM del modelo de prueba.

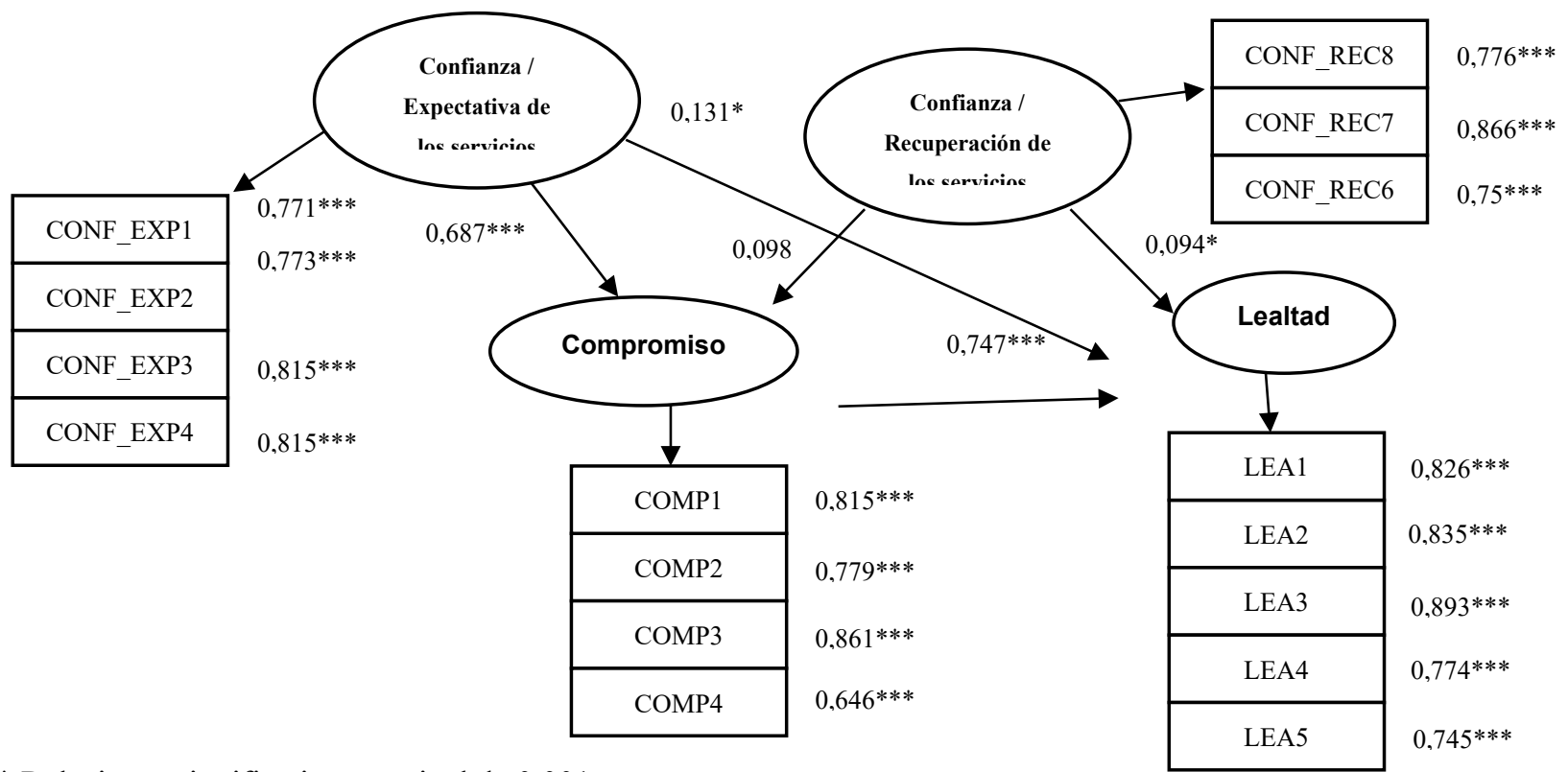

*** Relaciones significativas en nivel de 0,001

** Relaciones significativas en nivel de 0,01

* Relaciones significativas en nivel de 0,05

Fuente: Elaboración propia.

Según se puede visualizar en la Figura 2, de las 21 relaciones identificadas entre constructos/constructos y constructos/indicadores, 20 exhibieron significación estadística. Por lo tanto, es posible concluir que la validez nomológica fue alcanzada de forma parcial porque el constructo confianza/recuperación de los servicios no mostró significación estadística con el constructo consecuente compromiso.

En la Tabla 12, se presenta la descripción de las hipótesis actualizadas de este artículo con los respectivos resultados obtenidos en el modelo de prueba.

Tabla 12. Resumen de la constatación de hipótesis.

\begin{tabular}{|c|c|}
\hline Hipótesis & Resultado \\
\hline $\begin{array}{l}\text { H1_a: La confianza/expectativa de los servicios tiene un efecto significativamente positivo } \\
\text { sobre el compromiso. }\end{array}$ & Aceptada \\
\hline $\begin{array}{l}\text { H1_b: La confianza/recuperación de los servicios tiene un efecto significativamente positivo } \\
\text { sobre el compromiso. }\end{array}$ & Rechazada \\
\hline $\begin{array}{l}\text { H2_a: La confianza/expectativa de los servicios tiene un efecto significativamente positivo } \\
\text { sobre la lealtad. }\end{array}$ & Aceptada \\
\hline $\begin{array}{l}\text { H2_b: La confianza/recuperación de los servicios tiene un efecto significativamente positivo } \\
\text { sobre la lealtad. }\end{array}$ & Aceptada \\
\hline H3: El compromiso tiene un efecto significativamente positivo sobre la lealtad. & Aceptada \\
\hline
\end{tabular}

Fuente: Elaboración propia. 
Conforme se puede verificar, fueron propuestas inicialmente tres hipótesis que se convirtieron en cinco hipótesis durante el proceso de análisis estadístico. Así que, según la Tabla 12, cuatro hipótesis fueron aceptadas (H1_a, H2_a, H2_b y H3) y una hipótesis fue rechazada (H1_b).

En relación con la Varianza explicada del modelo, fueron calculados los valores de $\mathrm{R}^{2}$, según se puede visualizar en la Tabla 13, para todos los constructos que recibieron interferencia de otros constructos en el modelo de prueba.

Tabla 13. Varianza explicada de los constructos.

\begin{tabular}{lc}
\hline \multicolumn{1}{c}{ Constructo } & $\mathbf{R}^{2}-$ Modelo de prueba \\
\hline Confianza/expectativa de los servicios & No se aplica* \\
Confianza/recuperación de los servicios & No se aplica* \\
Compromiso & $57,7 \%$ \\
Lealtad & $83,1 \%$ \\
\hline
\end{tabular}

*No se aplica porque son constructos exógenos, es decir, no hay relación de constructos antecedentes a ellos. Fuente: Elaboración propia.

Según la Tabla 13 se puede verificar que el constructo lealtad presentó el mayor índice de explicación para el valor de $\mathrm{R}^{2}$, siendo un $83,1 \%$ para este modelo de prueba. Además, el constructo compromiso mostró un valor satisfactorio de $\mathrm{R}^{2}$ de un $57,7 \%$. En consonancia, la tesis doctoral de Breitenbach (2016) expuso que el constructo compromiso exhibió el valor de $\mathrm{R}^{2}$, igualmente satisfactorio en relación con el constructo retención y el artículo de Evanschitzky et al. (2006) concluyó que el compromiso impulsa la lealtad.

Aún en la Validez nomológica, específicamente en la SEM, se hace necesario llevar a cabo el cálculo del Índice de ajuste del modelo de prueba. Los índices elegidos para el presente estudio fueron: Chi-cuadrado $\left(X^{2}\right)$, Chi-cuadrado sobre grado de libertad (CMIN/DF), Índice de bondad del ajuste (GFI) e Índice ajustado de bondad de ajuste (AGFI), que son medidas de ajuste absoluto. Para el índice de mala calidad de ajuste fue el Error cuadrado de aproximación a las raíces medias (RMSEA), que también es una medida de ajuste absoluto (véase la Tabla 14).

Tabla 14. Índices de ajuste del modelo de prueba.

\begin{tabular}{ccc}
\hline Índice de ajuste & $\begin{array}{c}\text { Valor obtenido } \\
\text { para el modelo de } \\
\text { prueba }\end{array}$ & Valor idóneo \\
\hline$X^{2}$ & 449,133 & No definido \\
p-valor & 0,665 & $>0,05$ \\
DF & 98 & Lo mayor posible arriba de cero \\
CMIN/DF & 4,583 & $>1$ hasta 3 y para modelos más complejos \\
GFI & 0,922 & $\geq 0,90$ \\
AGFI & 0,892 & $\geq 0,90$ \\
RMSEA & 0,070 & $>0,03 \mathrm{y}<0,08$ \\
\hline
\end{tabular}

$X^{2}=$ Chi-cuadrado

$\mathrm{DF}=$ Grados de libertad

CMIN/DF = Chi-cuadrado sobre grado de libertad

GFI = Índice de bondad de ajuste

AGFI = Índice ajustado de bondad de ajuste

RMSEA = Error cuadrado de aproximación a las raíces medias

Fuente: Elaboración propia a partir de Hair et al. (2009) y Kline (2015). 
Según la Tabla 14, se puede verificar que todos los índices de ajuste presentaron valores satisfactorios, conforme rangos de valores idóneos en el modelo de prueba. Por consiguiente, los indicadores analizados exhibieron valores adecuados.

\section{Conclusiones y Recomendaciones.}

\subsection{Conclusiones del análisis empírico.}

Según los resultados empíricos obtenidos, se puede concluir que: (1) en el análisis descriptivo todos los ítems presentaron una media satisfactoria para los tres constructos investigados; (2) en el análisis factorial exploratorio dichos constructos exhibieron valores de KMO en nivel de excelencia; (3) en el análisis de fiabilidad de las escalas todos los constructos alcanzaron valores de alfa de Cronbach satisfactorios; (4) en la validez convergente, solo el constructo compromiso reveló un valor bajo en la AVE; (5) en la validez discriminante, la relación compromiso frente a lealtad ha sido la única en no presentar validez discriminante; (6) en la validez nomológica, el constructo confianza/recuperación de los servicios no mostró significación estadística con el constructo consecuente compromiso. Además, la utilización de dichos constructos en el modelo de prueba produjo un $\mathrm{R}^{2}$ de un $57,7 \%$ para el compromiso y un $83,1 \%$ para la lealtad, que son respectivamente positivos.

\subsection{Recomendaciones al Festival Rock in Rio.}

Teniendo en cuenta los hallazgos que ofrece este estudio, se presentan algunas recomendaciones que pueden ayudar a mejorar la gestión de la marca del RIR: (1) la deseada lealtad de marca debe partir de la adecuada gestión de las variables: confianza/expectativa de los servicios, confianza/recuperación de los servicios y compromiso. Así que las diferentes áreas de la empresa deben preocuparse por trabajar conectadas y en sintonía con los aspectos de la confianza de marca y del compromiso de marca, de lo contrario la lealtad de marca no será alcanzada y el modelo pierde su fin estratégico, limitándose al marketing tradicional y, por tanto, desperdiciando su mayor alcance de valor de marca; (2) la conquista de la confianza y del compromiso del cliente con la marca puede derivar en fuertes relaciones a largo plazo, lo que induce a los gestores a utilizar las estrategias del marketing relacional para fidelizar a sus clientes y alcanzar la lealtad de marca. Teniendo en cuenta todo lo mencionado, se recomienda que el RIR potencie la variable confianza por medio de acciones que cumplan las expectativas de los clientes y no les decepcionen. Por ejemplo, la decisión de irse, en determinado día al festival, está condicionada a la line-up de los conciertos, así que, si un determinado cantante cancela su actuación, como fue el caso de Lady Gaga en la edición de 2017, eso resulta en una insatisfacción que, ciertamente, podrá comprometer la confianza del cliente con la marca RIR; por tanto, se sugiere que este riesgo sea evitado. En relación con la variable compromiso, los diversos medios de comunicación social (Facebook, Instagram, Twitter, etc.), la página electrónica y el programa de fidelización son herramientas útiles para reforzar el compromiso del cliente con la marca RIR. Cada vez más, las empresas están estrechando sus relaciones con los clientes por medio de acciones constantes de comunicación personalizables. El RIR, por ejemplo, puede mantener una comunicación persistente con sus clientes, no solo en el año que se celebra el festival, por medio de una interacción individual (one to one). Por lo tanto, la lealtad de marca será una consecuencia de la buena gestión de la confianza y del compromiso.

\subsection{Contribución a la ciencia.}

Este trabajo de investigación logró comprender la relación existente de las variables confianza, compromiso y lealtad, desde una perspectiva de la marca del RIR. Su originalidad y su valor para la ciencia se basa en la falta de estudios similares en Brasil, en particular del sector del entretenimiento musical brasileño. En este sentido, esta investigación es un modesto estudio descriptivo que pretendió validar el modelo de prueba y que identificó que la escala de confianza necesita ser mejorada, pues ha sido dividida en dos constructos (confianza/expectativa de los servicios y confianza/recuperación de los servicios) que poseen validez de contenido, es decir, una división de los ítems conforme su sentido y lógica. Por lo tanto, este artículo aprovecha la oportunidad de probar teoría y modelo en una cultura 
diferente de las comúnmente usadas de los países desarrollados, que servirá para ulteriores estudios fructíferos sobre marcas.

\subsection{Limitaciones y futuras investigaciones.}

Esta investigación presenta una limitación sobre la posibilidad de realizar inferencias sobre la población, teniendo en cuenta que se trata de una muestra no probabilística y, por lo tanto, los resultados obtenidos no pueden ser utilizados para establecer inferencias poblacionales. Otra limitación relevante es que, aunque la academia científica ofrezca estudios de buena calidad acerca de las variables: confianza, compromiso y lealtad, hay que considerar que esas variables forman parte del universo tangible e intangible de las marcas y, por consiguiente, están en constantes evolución y están inducidas directamente por el ambiente de análisis y, sobre todo, por los continuos cambios del comportamiento del consumidor. La utilización del Facebook Ads para la recolección de los datos puede presentar limitaciones por el hecho de ser una herramienta de pago y generar una baja tasa de conversión dependiendo de la campaña publicitaria desarrollada. Por este motivo, cuanto mayor es la inversión financiera, mayor será el alcance de las personas impactadas con el enlace del cuestionario en línea, sin embargo, eso no es garantía de que éste sea cumplido en la misma proporción. Asimismo, el número de cuestionarios en línea descartados ha sido una limitación considerable. Por cierto, eso se justifica por el interés de las personas en concurrir a la recompensa ofrecida y no por el objeto del instrumento en sí. Además, se pedía el correo del encuestado para optar a la recompensa ofrecida, lo que puede haber estimulado respuestas más favorables a la marca de RIR, es decir, respuestas "políticamente correctas" para tener una mayor probabilidad de ganar el premio ofrecido. Por último, estudios futuros podrán buscar la constatación de las hipótesis en contextos diferentes del sector del entretenimiento musical brasileño. Incluso, su aplicación a otros países emergentes puede contribuir a la generalización de los resultados obtenidos. Asimismo, se pueden mejorar las escalas compromiso y Lealtad, buscándose mantener las relaciones presentes hasta el momento y al mismo tiempo buscar alcanzar la validez discriminante.

\section{Referencias}

Anuwichhanont, J. (2011). The impact of price perception on customer loyalty in the airline context. Journal of Business \& Economics Research, 9, 37-49.

Ballester, M.E.D. (2004). Applicability of a brand trust scale across product categories: a multigroup invariance analysis. European Journal of Marketing, 38(5/6), 573-592.

Ballester, M.E.D., \& Alemán, J.L.M. (2001). Brand trust in the context of consumer loyalty. European Journal of Marketing, 35, 1238-1258.

Ballester, M.E.D., \& Alemán, J.L.M. (2005). Does brand trust matter to brand equity? Journal of Product \& Brand Management, 14(3), 87-196.

Ballester, M.E.D., Alemán, J.L.M., \& Guillén, M.J.Y. (2003). Development and validation of a brand trust scale. International Journal of Market Research, 45, 335-353.

Bastos, D.H., Moura, L.R.C., \& Christino, J.M.M. (2015). Mensuração, teste e validação da confiança na marca. Revista Capital Científico-Eletrônica (RCCe), 13(2), 1-15.

Breitenbach, R. (2016). Táticas vinculantes, reputação do provedor de serviços, confiança e compromisso como antecedentes da retenção de clientes. Caxias do Sul, 2016. Tesis Doctoral (Doctorado en Administración) - Universidade de Caxias do Sul, Rio Grande do Sul, Brasil.

Carneiro, L.F. (2011). Rock in Rio: a História do Maior Festival de Música do Mundo. Rio de Janeiro: 
Editora Globo.

Chaudhuri, A., \& Holbrook, B.M. (2001). The chain of effects from brand trust and brand affects to brand performance; the role of brand loyalty. Journal of Marketing, 65(2), 81-93.

Churchill Jr., G.A. (1999). Marketing research: methodological foundations. Fort Laudardale: Harcourt College Publishers.

Colmenares, O., \& Saavedra, J. (2007). Aproximación teórica de la lealtad de marca: enfoques y valoraciones. Cuadernos de Gestión, 7(2), 69-81.

Doorn, J.V., Lemon, K.N., Mittal, V., Nass, S., Pick, D., Pirner, P., \& Verhoef, P.C. (2010). Customer Engagement Behavior: theoretical foundations and research directions. Journal of Service Research, 13, (3), 253-266.

Ercis, A., Ünal, S., Candan, F.B., \& Yildirim, H. (2012). The effect of brand satisfaction, trust and brand commitment on Loyalty and repurchase intentions. Journal of Procedia - Social and Behavioral Sciences, 58, 395-1404.

Evanschitzky, H., Iyer, G.R., Plassmann, H., Niessing, J., \& Meffert, H. (2006). The relative strength of affective commitment in securing loyalty in service relationships. Journal of Business Research, 59, 1207-1213.

Frederico, E. (2004). Avaliação dos antecedentes e das consequências da confiança nas relações entre varejistas e fabricantes do mercado de vestuário. São Paulo, 2004. Tesis Doctoral (Doctorado en Administración) - Fundação Getúlio Vargas, São Paulo, Brasil.

Fullerton, G. (2003). When does commitment lead to loyalty? Journal of Service Research, 5(4), 33-44.

Fullerton, G. (2014). The moderating effect of normative commitment on the service quality-customer retention relationship. European Journal of Marketing, 48, (3/4) 657-673.

Guede, J.R.S. (2014). Marketing de relaciones en los medios sociales: estudio empírico de los antecedentes y consecuentes de la calidad de la relación en los sitios web de viajes. Tesis Doctoral (Doctorado en Economía de la Empresa) - Universidad Rey Juan Carlos, Madrid, España.

Guo, L., Gruen, T.W., \& Tang, C. (2015). Seeing relationships the lens of psychological contracts: the structure of consumer service relationships. Journal of the Academy of Marketing Science, 45, 357-376.

Hair, J.F.J., Black, W.C., Babin, B.J., Anderson, R.E., \& Tatham, R.L. (2009). Análise multivariada de dados. 6. ed. Porto Alegre: Bookman.

Hair, J.F.J., Wolfinbarger, M., Ortinau, D.J., \& Bush, R.P. (2010). Fundamentos de pesquisa de marketing. Porto Alegre: Bookman.

Hanzaee, K.H., \& Andervazh, L. (2012). An analysis of some moderating variables on the value, brand trust and brand loyalty chain. Research Journal Applied Sciences, Engineering and Technology, 4, 1403-1413.

Henning-Thurau, T., Gwinner, K.P., \& Gremler, D.D. (2002). Understanding relationship marketing 
outcomes an integration of relational benefits and relationship quality. Journal of Service Research, 4(3), 230-247.

Higgins, E.T. (2006). Value from Hedonic Experience and Engagement. Psychological Review, 113, 439-460.

Höpner, A. (2017). Construção da experiência de consumo: um olhar para compreender o valor nas experiências. Porto Alegre, 2017. Tesis Doctoral (Doctorado en Administración) - Pontifícia Universidade Católica do Rio Grande do Sul, Porto Alegre, Brasil.

IBGE - Instituto Brasileiro de Geografia e Estatística. (2018). Portal Brasil - Panorama. Rio de Janeiro: IBGE.

Kazemi, A., Paemami, V.M., Abbaszadeh, A., \& Pourzamani, J. (2013). Impact of Brand Identity on Customer Loyalty and Word of Mouth Communications, Considering Mediating Role Customer Satisfaction and Brand Commitment. (Case Study: Customers of Mellat Bank in Kermanshah). International Journal of Academic Research in Economics and Management Sciences, 2(4), 1-14.

Kemp, E., \& Bui, M. (2011). Healthy brands: establishing brand credibility, commitment and connection among consumers. Journal of Consumer Marketing, 28(6), 429-437.

Kim, J., Morris, J.D., \& Swait, J. (2008). Antecedents of true brand loyalty. Journal of Advertising, 3(2), 99-117.

Kline, R.B. (2015). Principles and practice of structural equation modeling. Guilford publications.

Koetz, C.I., Terres, M.S., Santos, C.P., \& Caten, C.S. (2009). O Papel da Confiança na Marca na Intenção de Adoção de Novas Tecnologias em Trocas Business-to-Consumer. In: XXXIII Encontro Nacional dos Programas de Pós-Graduação em Administração (ENANPAD). São Paulo.

Lee, D., Moon, J., Kim, Y.J., \& Yi, M.Y. (2015). Antecedents and consequences of mobile phone usability: linking simplicity and interactivity to satisfaction, trust, and brand loyalty. Information \& Management - Elsevier, 52, 295-304.

Malhotra, N.K. (2010). Pesquisa de Marketing: uma orientação aplicada. 6.ed. Porto Alegre: Bookman.

Malhotra, N.K., Birks, D., \& Wills, P. (2012). Marketing research: applied approach. 4.ed. New York: Person.

Mathew, V., Thomas, S., \& Injodey, J. (2012). Direct and Indirect Effect of Brand Credibility, Brand Commitment and Loyalty Intention on Brand Equity. Economic Review, 10, 73-82.

Matzler, K., Bidmon, S., \& Grabner-Kräuter, S. (2006). Individual determinants of brand affect: the role of the personality traits of extraversion and openness to experience. Journal of Product \& Brand Management, 15, 427-434.

Matzler, K., Grabner-Kräuter, S., \& Bidmon, S. (2008). Risk aversion and brand loyalty: The mediating role of brand trust and brand affect. Journal of Product \& Brand Management, 17, 154-162.

Moorman, C., Zaltman, G., \& Deshpande, R. (1992). Relationships between providers and users of 
marketing research: the dynamics of trust within and between organizations. Journal of Marketing Research, 29, 314-329.

Morgan, R.M., \& Hunt, S.D. (1994). The commitment-trust theory of relationship marketing. Journal of Marketing, 58(3), 20-38.

Moura, L.R.C. (2010). Um estudo comparativo de teorias da ação e suas extensões para explicar a tentativa de perder peso. Belo Horizonte, 2010. Tesis Doctoral (Doctorado em Administración) - Universidade Federal de Minas Gerais, Minas Gerais, Brasil.

Oliver, R.L. (1999). Whence customer loyalty. Journal of Marketing, 63, 33-44.

Palmatier, R.W. (2008). Interfirm Relational Drivers of Customer Value. Journal of Marketing, 72, 7689.

Palmatier, R.W., Dant, R.P., Grewal, D., \& Evan, K.R. (2006). Factors influencing the effectiveness of relationship marketing: a meta-analysis. Journal of Marketing, 70(4), 136-153.

Palmatier, R.W., Houston, M.B., Dant, R.P., \& Grewal, D. (2013). Relationship velocity: toward a theory of relationship dynamics. Journal of Marketing, 77(1), 13-30.

Ponder, N., Holloway, B.B., \& Hansen, J.D. (2016). The mediating effects of customers intimacy perceptions on the trust-commitment relationship. Journal of Services Marketing, 3(1), 75-87.

Rock In Rio (2017, 21 julio). Nova Cidade do Rock. Descargado de: http://rockinrio.com/rio/pt-BR>.

Rock World (2017). Rock in Rio. Departamento de marketing. Informaciones concedidas por Mariana Léllis. Rio de Janeiro, 2017.

Rodrigues, P.M.A. (2015). Influencia de la experiencia de marca, en el valor de la marca, por vía de la satisfacción y lealtad de clientes. Tesis Doctoral (Doctorado en Economía de la Empresa) Universidad Rey Juan Carlos, Madrid, España.

Sahin, A., Zehir, C., \& Kitapçi, H. (2011). The Effects of Brand Experiences, Trust and Satisfaction on Building Brand Loyalty; An Empirical Research On Global Brands. 7th International Strategic Management Conference, p.1288-1301.

Sallam, M. A. (2015). The effects of brand credibility on customers wom communication: the mediating role of brand commitment. European Journal of Business and Social Sciences, 4(9), 164-176.

Sampieri, R.H., Collado, C.F., \& Lucio, P.B. (2006). Metodología de la investigación. 4.ed. Mexico: MacGraw-Hill.

Silva, M.A., \& Tincani, D.P. (2013). Características e componentes do marketing de experiências: análise das ações realizadas pelo Itaú Unibanco no Rock in Rio 2011. Revista Científica Eletrônica UNISEB, 1(2), 147-161.

Sung, Y., \& Kim, J. (2008). Effects of brand personality on brand trust and brand affect. Psychology \& Marketing, 27, 639-661. 